\title{
Author Correction: Dysregulated ribonucleoprotein granules promote cardiomyopathy in RBM20 gene-edited pigs
}

\author{
Jay W. Schneider (D), Saji Oommen (D), Muhammad Y. Qureshi D, Sean C. Goetsch, David R. Pease (D), \\ Rhianna S. Sundsbak, Wei Guo (D), Mingming Sun (D), Han Sun D, Hidehito Kuroyanagi, Dennis A. Webster, \\ Alexander W. Coutts, Kimberly A. Holst, Brooks S. Edwards, Nikolas Newville, Matthew A. Hathcock, Tamene Melkamu, \\ Francesca Briganti (D), Wu Wei (D), Maria G. Romanelli D, Scott C. Fahrenkrug, Doug E. Frantz (D), Timothy M. Olson, \\ Lars M. Steinmetz (D), Daniel F. Carlson (D), Timothy J. Nelson and Wanek Program Preclinical Pipeline*
}

Correction to: Nature Medicine https://doi.org/10.1038/s41591-020-1087-x, published online 13 November 2020.

In the version of this article initially published, the labels (all 'WT') along the horizontal axis were incorrect for the middle and right bars in both plots in Fig. 1k. All correct labels are (left to right in each plot): WT, HTZ, HMZ. Also, the $n$ value for the far right bar in the right plot $(n=6)$ is incorrect. The correct value is ' $n=5$ '. The errors have been corrected in the HTML and PDF versions of the article.

${ }^{\star}$ A list of authors and their affiliations appears online.

Published online: 2 June 2021

https://doi.org/10.1038/s41591-021-01412-8

( ) The Author(s), under exclusive licence to Springer Nature America, Inc. 2021

\section{Author Correction: Systematic screening identifies therapeutic antisense oligonucleotides for Hutchinson-Gilford progeria syndrome}

\begin{abstract}
Madaiah Puttaraju, Michaela Jackson, Stephanie Klein, Asaf Shilo, C. Frank Bennett, Leslie Gordon, Frank Rigo and Tom Misteli iD
\end{abstract}

Correction to: Nature Medicine https://doi.org/10.1038/s41591-021-01262-4, published online 11 March 2021.

In the version of this article initially published, the source of lonafarnib was not provided. This should be included as the second sentence of the Acknowledgements section, as follows: "Lonafarnib was provided by Merck through The Progeria Research Foundation's Pre-Clinical Drug Supply Program.” The error has been corrected in the HTML and PDF versions of the article.

Published online: 1 June 2021

https://doi.org/10.1038/s41591-021-01415-5

(C) The Author(s), under exclusive licence to Springer Nature America, Inc. 2021

\section{Author Correction: Delayed production of neutralizing antibodies correlates with fatal COVID-19}

Carolina Lucas (D), Jon Klein (1), Maria E. Sundaram, Feimei Liu(1), Patrick Wong, Julio Silva (D), Tianyang Mao (D), Ji Eun Oh (D), Subhasis Mohanty, Jiefang Huang, Maria Tokuyama, Peiwen Lu®i, Arvind Venkataraman, Annsea Park, Benjamin Israelow (1), Chantal B. F. Vogels (D), M. Catherine Muenker (D), C-Hong Chang, Arnau Casanovas-Massana, Adam J. Moore, Joseph Zell, John B. Fournier, Yale IMPACT Research Team ${ }^{\star}$, Anne L. Wyllie $\mathbb{B}$, Melissa Campbell,

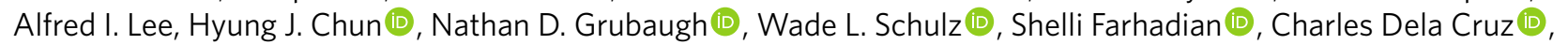

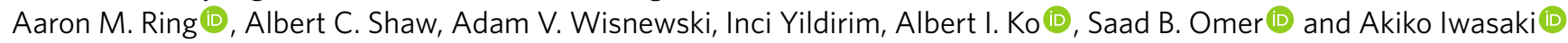

Correction to: Nature Medicine https://doi.org/10.1038/s41591-021-01355-0, published online 5 May 2021.

In the version of this article initially published, two author names (Alfred Lee and Saad Omer) were missing the middle initial. The correct names are 'Alfred I. Lee' and 'Saad B. Omer' (respectively). The errors have been corrected in the HTML and PDF versions of the article.

${ }^{*}$ A list of authors and their affiliations appears online.

Published online: 18 June 2021

https://doi.org/10.1038/s41591-021-01416-4

(c) The Author(s), under exclusive licence to Springer Nature America, Inc. 2021 